\title{
Parameterized nonrelativistic limit of stellar structure equations in Ricci-based gravity theories
}

\author{
Gonzalo J. Olmo, ${ }^{1,2, *}$ Diego Rubiera-Garcia ${ }^{3, \dagger}$ and Aneta Wojnar ${ }^{4, \$}$ \\ ${ }^{1}$ Departamento de Física Teórica and IFIC, Centro Mixto Universidad de Valencia-CSIC. Universidad \\ de Valencia, Burjassot-46100, Valencia, Spain \\ ${ }^{2}$ Departamento de Física, Universidade Federal da Paraíba, 58051-900 João Pessoa, Paraíba, Brazil \\ ${ }^{3}$ Departamento de Física Teórica and IPARCOS, Universidad Complutense de Madrid, \\ E-28040 Madrid, Spain \\ ${ }^{4}$ Laboratory of Theoretical Physics, Institute of Physics, University of Tartu, \\ W. Ostwaldi 1, 50411 Tartu, Estonia
}

(Received 5 May 2021; accepted 30 June 2021; published 16 July 2021)

\begin{abstract}
We present the nonrelativistic limit of the stellar structure equations of Ricci-based gravities, a family of metric-affine theories whose Lagrangian is built via contractions of the metric with the Ricci tensor of an a priori independent connection. We find that this limit is characterized by four parameters that arise in the expansion of several geometric quantities in powers of the stress-energy tensor of the matter fields. We discuss the relevance of this result for the phenomenology of nonrelativistic stars, such as main-sequence stars as well as several substellar objects.
\end{abstract}

DOI: 10.1103/PhysRevD.104.024045

\section{INTRODUCTION}

In the last few years our community has witnessed the success of Einstein's general theory of relativity (GR) when confronted with a new pool of astrophysical data: from the LIGO and VIRGO observations of gravitational waves out of binary mergers $[1,2]$, to the radio measurements by the EHT of the plasma around the supermassive central object of the M87 galaxy [3], both of which are compatible with the predictions of GR on the properties of compact objects (black holes and neutron stars). Together with solar system experiments [4], and the compatibility of the concordance cosmological model with observations [5], this bunch of predictions have underpinned our trust on the reliability of GR to describe a wide range of gravitational phenomena. However, at the same time, the exploration of the strong-field regime by these new probes also enables us to test alternative descriptions of the gravitational fieldcommonly known as modified theories of gravity-via their accompanying phenomenology. For some basic references on these theories see, e.g., [6-12].

In addition to black holes and their observational discriminators in terms of gravitational waves [13-17] and lensing of electromagnetic radiation [18-23], the astrophysics of stellar objects represents another promising playground to test the viability of these modified theories of gravity, thanks to the availability of numerous data from

\footnotetext{
*gonzalo.olmo@uv.es

drubiera@ucm.es

¥aneta.magdalena.wojnar@ut.ee
}

different probes [24]. From a theoretical point of view, stellar objects are usually split into two classes: relativistic stars such as neutron stars, where the full power of the Einstein field equations (via the Tolman-OppenheimerVolkoff equation) must be called upon [25]; and nonrelativistic stars, to which main-sequence stars as well as brown and red dwarfs and giant exoplanets [26] belong, ${ }^{1}$ where one can neglect the contribution of the pressure of the matter fields as compared to their energy density.

As opposed to neutron stars, where the uncertainty of the equation of state at supranuclear densities is entangled with the new parameter(s) of the modified gravity side (for a review of this issue see [28]), nonrelativistic stars may offer a cleaner scenario to test modifications to GR predictions since they are more weakly dependent on nongravitational physics than their relativistic counterparts. This is of particular relevance for those models in which the new physics appears as a modification of the Poisson equation inside astrophysical bodies [29]. Despite the fact that numerous additional aspects of the modeling of such stars (including thermodynamics, description of their atmospheres, energy generation rates, etc.) are needed in order to achieve a realistic description of their structure, simple analytical models are capable to provide reasonable estimates to some global properties of such nonrelativistic stars. Examples of this are the limiting masses, such as the

\footnotetext{
${ }^{1}$ While white dwarfs are typically classified as nonrelativistic objects, relativistic effects need to be incorporated to reliably describe certain aspects of the physics of these stars [27].
} 
Chandrasekhar mass for white dwarfs [30-35] or the minimum required mass for a star to stably burn hydrogen [36-38] and deuterium [39], but it may also have a nonnegligible impact in the description of the early evolution of low-mass stars [40], in the cooling process of brown dwarfs [41], and in age-estimation techniques such as those based on the lithium depletion method [42].

The main aim of the present paper is to work out the nonrelativistic limit of the stellar structure equations corresponding to a large family of metric-affine theories of gravity, where metric and affine connection are regarded as independent entities [43]. More specifically, our target are those theories built as scalar functions of the metric and the Ricci tensor of the affine connection (Ricci-based gravities, or RBGs for short [44]). The interest in this family of theories lies on the fact that their vacuum dynamics is exactly that of GR (plus a cosmological constant term, which is usually neglected in stellar structure models), which allows (most of) them to automatically pass both solar system experiments and gravitational wave observations so far, while the nontrivial role played by the connection induces new nonlinearities engendered by the matter fields [45]. This has important consequences for the predictions of these theories at several levels, including the resolution of space-time singularities inside black holes [46] and in early cosmology [47], the existence of new observable effects in 4-fermion contact interactions from quantum fields [48], or the development of new nontrivial effective interactions $[49,50]$.

Regarding the description of stellar structure, RBGs are capable to modify the mass-radius relations of neutron stars, which allows to raise their maximum mass with realistic equation of state to match observations of neutron stars above two solar masses [51]; and also alter the Chandrasekhar's limit of white dwarfs, which might allow to address the existence of super-Chandrasekhar white dwarfs, whose masses are suggested to be up to two times the standard Chandrasekhar limit [52-55] (see chapter 3 of [28] for details on these issues). Moreover, the nonrelativistic limits of some RBGs have been recently employed to work out the minimum main sequence mass of quadratic $f(R)$ gravity [56] and or the minimum deuterium burning mass for Eddington-inspired Born-Infeld gravity (EiBI, [39]), where the current modeling of these scenarios and their compatibility with observations still leaves some room for modifications of GR, becoming a source of strong constraints on the parameters of RBGs. Furthermore, studies related to low-mass stars in Palatini $f(R)$ gravity $[40,42]$ demonstrated that RBGs could also provide an explanation of the discrepancy between predicted and dynamical masses of the $\mathrm{M}$ dwarfs and pre-main sequence stars with masses below 0.5 solar masses $[57,58]$.

In this work we shall show that the nonrelativistic limit of the whole RBG family can be fully characterized in terms of four parameters associated to the expansion in powers of the stress-energy tensor of the matter fields, two of which belong to the shape of the RBG Lagrangian density, and the other two to the deformation matrix that relates the Einstein and RBG frame metrics of the corresponding field equations. From the general expression that we obtain, those of $f(R)$ and EiBI gravity are easily obtained, which provides a consistency check of our results. We shall finally discuss some prospects for applications of this nonrelativistic limit of RBGs inside astrophysical bodies.

\section{RICCI-BASED GRAVITY THEORIES}

\section{A. Action and field equations}

The class of RBG theories is defined by the action

$$
\mathcal{S}=\int d^{4} x \sqrt{-g} \mathcal{L}_{G}\left(g_{\mu \nu}, R_{\mu \nu}\right)+\mathcal{S}_{m}\left(g_{\mu \nu}, \psi_{m}\right),
$$

where $g$ is the determinant of the space-time metric $g_{\mu \nu}$, the Ricci tensor $R_{\mu \nu}$ is built out solely with the affine connection $\Gamma \equiv \Gamma_{\mu \nu}^{\lambda}$, which is independent of the metric (Palatini or metric-affine approach), while the gravitational Lagrangian $\mathcal{L}_{G}$ is a scalar function built out of powers of traces of the object $M^{\mu}{ }_{\nu} \equiv g^{\mu \alpha} R_{\alpha \nu}$. As for the matter sector, $\mathcal{S}_{m}=\int d^{4} x \sqrt{-g} \mathcal{L}_{m}\left(g_{\mu \nu}, \psi_{m}\right)$, it is assumed to be minimally coupled to the metric, with $\psi_{m}$ denoting collectively the matter fields. In this action we have neglected the role of torsion (the antisymmetric part of the connection), since for minimally coupled bosonic fields it trivializes [59], and the antisymmetric part of the Ricci tensor, to safeguard the theory against potential ghostlike instabilities [60,61]. Defined this way, the RBG family includes some wellknown theories, such as GR itself, $f(R)$ gravity, quadratic gravity, as well as EiBI gravity and many of its extensions [11].

It has been shown elsewhere (see for instance Ref. [11]) that the field equations of the action (1) admit a representation of the form

$$
G_{\nu}^{\mu}(q)=\frac{\kappa^{2}}{|\hat{\Omega}|^{1 / 2}}\left(T^{\mu}{ }_{\nu}-\delta_{\nu}^{\mu}\left(\mathcal{L}_{G}+\frac{T}{2}\right)\right)
$$

where $T_{\mu \nu}=-\frac{2}{\sqrt{-g}} \frac{\partial \mathcal{L}_{m}}{\partial g^{m \nu}}$ is the energy-momentum tensor of the matter fields with $T$ its trace, while $G^{\mu}{ }_{\nu}(q)$ is the Einstein tensor associated to a new rank-two tensor $q_{\mu \nu}$ such that the connection $\Gamma$ is Levi-Civita of it, that is, $\nabla_{\mu}^{\Gamma}\left(\sqrt{-q} q^{\alpha \beta}\right)=0$. For any RBG this tensor is related to the space-time metric $g_{\mu \nu}$ via the fundamental relation

$$
q_{\mu \nu}=g_{\mu \alpha} \Omega^{\alpha}{ }_{\nu},
$$

where the deformation matrix $\Omega^{\alpha}{ }_{\nu}$ (whose determinant is denoted by vertical bars) depends on the particular $\mathcal{L}_{G}$ 
chosen, but it can always be expressed on-shell (as so does $\mathcal{L}_{G}$ itself) as a function of the matter fields and (possibly) the space-time metric too. The representation (2) also reinforces the fact that the RBG field equations are second-order and that in vacuum, $T^{\mu}{ }_{\nu}=0$, these equations and their solutions boil down to their GR counterparts. Therefore, no extra degrees of freedom (beyond the usual two polarizations of the gravitational field) are propagated in these theories.

\section{B. The two frames of RBGs and the mapping equations}

The resemblance of the representation (2) with the usual GR field equations strongly suggests that an Einstein frame should also be possible, that is [62]

$$
G_{\nu}^{\mu}(q)=\kappa^{2} \tilde{T}^{\mu}{ }_{\nu}(q),
$$

where $\tilde{T}^{\mu}{ }_{\nu}(q)$ would be the energy-momentum tensor of some new matter Lagrangian density $\tilde{\mathcal{L}}_{m}\left(q_{\mu \nu}, \tilde{\psi}_{m}\right)$, built of the same type of fields as the original $T^{\mu}{ }_{\nu}(g)$, but with a different functional dependence. For this representation to work, one needs to systematically remove all dependencies on the space-time metric $g_{\mu \nu}$ in favor of those of $q_{\mu \nu}$. This intuitive idea was given explicit support and internal consistence in a series of works by some of us $[44,63,64]$. The most general case for the matter sector studied so far considers an anisotropic fluid (which is suitable for stellar structure modeling), whose energy-momentum tensor can be written, in terms of the space-time metric, as

$T^{\mu}{ }_{\nu}(g)=\left(\rho+p_{\perp}\right) u^{\mu} u_{\nu}+p_{\perp} \delta_{\nu}^{\mu}+\left(p_{r}-p_{\perp}\right) \chi^{\mu} \chi_{\nu}$,

where $\rho$ is the energy density, and $p_{r}$ and $p_{\perp}$ the radial and tangential pressures, respectively, and we have introduced the unit timelike $g^{\mu \nu} u_{\mu} u_{\nu}=-1$ and spacelike $g^{\mu \nu} \chi_{\mu} \chi_{\nu}=$ +1 vectors, respectively. Note that in a comoving frame this energy-momentum tensor can simply be expressed as $T^{\mu}{ }_{\nu}=\operatorname{diag}\left(-\rho, p_{r}, p_{\perp}, p_{\perp}\right)$.

In the GR frame, the corresponding energy-momentum tensor reads formally the same

$\tilde{T}^{\mu}{ }_{\nu}(q)=\left(\tilde{\rho}+\tilde{p}_{\perp}\right) v^{\mu} v_{v}+\tilde{p}_{\perp} \delta_{\nu}^{\mu}+\left(\tilde{p}_{r}-\tilde{p}_{\perp}\right) \xi^{\mu} \xi_{v}$,

with new energy density $\tilde{\rho}$ and radial $\tilde{p}_{r}$ and tangential $\tilde{p}_{\perp}$ pressures, and unit timelike $q^{\mu \nu} v_{\mu} v_{\nu}=-1$ and spacelike $q^{\mu \nu} \xi_{\mu} \xi_{\nu}=+1$ vectors. Comparing the representations (2) and (4) of the field equations one finds that the energy density and pressures of the two fluids must be related as

$$
\begin{gathered}
\tilde{p}_{\perp}=\frac{1}{|\hat{\Omega}|^{1 / 2}}\left[\frac{\rho-p_{r}}{2}-\mathcal{L}_{G}\right] \\
\tilde{\rho}+\tilde{p}_{\perp}=\frac{\rho+p_{\perp}}{|\hat{\Omega}|^{1 / 2}}
\end{gathered}
$$

$$
\tilde{p}_{r}-\tilde{p}_{\perp}=\frac{p_{r}-p_{\perp}}{|\hat{\Omega}|^{1 / 2}}
$$

which shall be called hereafter the mapping equations between the two frames. Given that the determinant of the deformation matrix, $|\hat{\Omega}| \equiv|\hat{\Omega}|\left(T^{\mu}{ }_{\nu}, g_{\mu \nu}\right)$, is a function of $\rho$, $p_{r}$, and $p_{\perp}$, these equations establish a correspondence between GR coupled to some anisotropic fluid (tilded variables) and a given RBG coupled to another anisotropic fluid (untilded variables). Moreover, this correspondence can be used to obtain solutions of the latter starting from a seed solution of the former using purely algebraic transformations, as explicitly verified in Refs. [63,64] with concrete examples. Besides their obvious interest for the sake of finding analytic solutions of physical interest of modified gravity [65], the mapping equations also allow us to carry out formal manipulations to handle specific systems, as the one of (the newtonian limit of) stellar structure considered in this work.

\section{PARAMETERIZED NONRELATIVISTIC LIMIT OF RBGS}

For the analysis of nonrelativistic stars (and relativistic stars alike) one typically neglects anisotropies, whose estimated effects in realistic scenarios are (assumed to be) smaller than other degeneracies/unknowns (see however [66]). Thus we can set $p_{\perp}=p_{r}=p$ and $\tilde{p}_{\perp}=\tilde{p}_{r}=\tilde{p}$ to obtain a perfect fluid on both frames:

$$
\begin{aligned}
& {T^{\mu}{ }_{\nu}}=(\rho+p) u^{\mu} u_{\nu}+p \delta_{\nu}^{\mu} \\
& \tilde{T}_{\nu}^{\mu}=(\tilde{\rho}+\tilde{p}) v^{\mu} v_{v}+\tilde{p} \delta_{\nu}^{\mu},
\end{aligned}
$$

such that the mapping equations (7), (8), and (9) boil down to

$$
\begin{gathered}
\tilde{p}=\frac{1}{|\Omega|^{1 / 2}}\left[\frac{\rho-p}{2}-\mathcal{L}_{G}\right] \\
\tilde{\rho}+\tilde{p}=\frac{\rho+p}{|\Omega|^{1 / 2}} .
\end{gathered}
$$

In order to proceed further, we must find a representation of the deformation matrix $\Omega^{\mu}{ }_{\nu}$. Since this quantity is a nonlinear function of the energy-momentum tensor, it can be formally written as

$$
\Omega^{\mu}{ }_{\nu}=\delta_{\nu}^{\mu}+\sum_{n=1}^{\infty} \sum_{i_{n}=0}^{3} c_{\left(n, i_{n}\right)}\left(T^{\left(n, i_{n}\right)}\right)^{\mu}{ }_{\nu}
$$

where the $\left(T^{\left(n, i_{n}\right)}\right)^{\mu}{ }_{\nu}$ represent tensor structures that can be built with $n$ powers of the energy-momentum tensor [50]. The zeroth order term $\delta_{\nu}^{\mu}$ simply indicates that in the lowenergy limit one recovers the GR dynamics. This form of 
the matrix $\Omega^{\mu}{ }_{\nu}$ is the most reasonable scenario compatible with Lorentz covariance, and other possibilities are seemingly pathological [67]. Given the simplicity of (12) and (13), it is easy to see that these infinite sums must boil down to

$$
\Omega^{\mu}{ }_{\nu}=A \delta_{\nu}^{\mu}+B u^{\mu} u_{\nu}
$$

using the RBG frame variables, with $A$ and $B$ being some functions of $\rho$ and $p$, or to

$$
\Omega^{\mu}{ }_{\nu}=\tilde{A} \delta_{\nu}^{\mu}+\tilde{B} v^{\mu} v_{\nu}
$$

if we employ the GR frame variables instead, with $\tilde{A}$ and $\tilde{B}$ some functions of $\tilde{\rho}$ and $\tilde{p}$. Focusing on the zeroth and first order contributions, which will be the dominant terms in a perturbative expansion, we get

$$
\begin{aligned}
\Omega^{\mu}{ }_{\nu} & \approx \delta_{\nu}^{\mu}+\alpha T \delta_{\nu}^{\mu}+\beta T_{\nu}^{\mu} \\
& =(1+\alpha(3 p-\rho)+\beta p) \delta_{\nu}^{\mu}+\beta(\rho+p) u^{\mu} u_{\nu},
\end{aligned}
$$

where we have made use of the equality $T=-\rho+3 p$ and suitably rearranged terms. One should note that the coefficients $\alpha$ and $\beta$ must have dimensions of inverse density, such that we could write them as $\alpha=\tilde{\alpha} / \rho_{0}$ and $\beta=\tilde{\beta} / \rho_{0}$, with $\rho_{0}$ some density scale. According to the analysis of [50], this scale could be as high as $\rho_{0} \sim 10^{25} \mathrm{~g} / \mathrm{cm}^{3}$, which is well above the density of nuclear matter, $\sim 10^{14} \mathrm{~g} / \mathrm{cm}^{3}$, and of the interior of any known star. Since this bound has been derived in a very particular context involving light-bylight scattering experiments in particle accelerators, for our phenomenological purposes we will simply assume that $\rho_{0}$ is large enough so that the correcting terms associated to it can be considered as small perturbations, making unnecessary the consideration of higher-order terms in the perturbative expansion.

In the nonrelativistic limit, $p \ll \rho$, the components of the matrix (17) read explicitly as $(i=1,2,3)$

$$
\Omega^{t}{ }_{t}=1-(\alpha+\beta) \rho ; \quad \Omega^{i}{ }_{i}=1-\alpha \rho,
$$

and hereafter we shall refer to this limit and neglect contributions in $p$ (unless explicitly stated) as compared to those in $\rho$ (at the same order). Since this matrix is diagonal, the computation of the determinant $|\hat{\Omega}|$ is straightforward:

$$
|\Omega|^{1 / 2}=\left(\Omega_{t}^{t}\right)^{1 / 2}\left(\Omega_{i}^{i}\right)^{3 / 2}=1-\left(2 \alpha+\frac{\beta}{2}\right) \rho .
$$

Let us point out that similar expressions for $\Omega^{\mu}{ }_{\nu}$ and its determinant can be written in the GR frame (tilted variables) just by adding tildes to all the constants and functions. Then the mapping equations (12) and (13) will provide the correspondence between the functions in both representations. Using one of them or the other is thus just a matter of convenience for each specific computation, though at the end of the day the physical result has to be written in terms of the physical variables (that is, the untilded ones).

Now we need to find a similar expansion for the gravitational Lagrangian $\mathcal{L}_{G}$. Since it is a scalar quantity, in general, it can be expanded as an infinite series of traces of the objects $\left(T^{\left(n, i_{n}\right)}\right)^{\mu}{ }_{\nu}$. Likewise for $\Omega^{\mu}{ }_{\nu}$, retaining terms up to quadratic order in this series yields the result ${ }^{2}$

$$
\mathcal{L}_{G}=-\frac{1}{2 \kappa^{2}}\left(\kappa^{2} T+\gamma T_{\alpha}^{\mu} T_{\mu}^{\alpha}+\delta T^{2}\right)
$$

where we have inserted a factor $\kappa^{2}$ in the first term to identify it as the GR relation between the curvature scalar and the trace of the energy-momentum tensor. The other two terms yield the RBG corrections at quadratic order in the energy-momentum tensor and its trace, where we stress that for dimensional consistency the parameters $(\gamma, \delta)$ must have implicit $\kappa^{4}$ factors. For the perfect fluid energymomentum tensor (10) this expression becomes, for nonrelativistic fields:

$$
\mathcal{L}_{G} \approx \frac{\rho-3 p}{2}-\frac{(\gamma+\delta) \rho^{2}}{2 \kappa^{2}}
$$

where for the moment we have kept the term linear in $p$ but neglected one in $\rho \cdot p$ and another in $p^{2}$, as they are assumed to be smaller than the one quadratic in $\rho$.

Having under control the main objects on the righthand-side of the mapping equations (12) and (13), the next step in our analysis is to cast the field equations for stellar structure (the TOV equations). From the GR representation (4), such equations are found from the conservation of the energy-momentum tensor (as follows from the fulfillment of Bianchi's identities in (4), $\nabla_{\mu} \tilde{T}^{\mu}{ }_{\nu}(q)=0$, which, for a static, spherically symmetric metric, $d s_{q}^{2}=$ $q_{t t} d t^{2}+q_{x x} d x^{2}+x^{2} d \Omega^{2}$, yields

$$
\tilde{p}_{x}=-(\tilde{\rho}+\tilde{p}) \frac{q^{t t}}{2} \partial_{x} q_{t t} .
$$

Furthermore, by assuming this line element to take the form

$$
d s_{q}^{2}=-C(x) e^{\psi(x)} d t^{2}+\frac{d x^{2}}{C(x)}+x^{2} d \Omega^{2},
$$

and introducing the standard mass ansatz $C(x)=$ $1-2 M(x) / x$, then the functions $\{\psi(x), M(x)\}$ can be determined by resolution of the RBG field equations (4).

\footnotetext{
${ }^{2}$ We are neglecting here the zeroth-order term, which would correspond to a cosmological constant that does not contribute significantly to stellar quantities.
} 
Such a resolution parallels the analog problem in GR, and yields the well-known result

$$
\tilde{p}_{x}=-\frac{(\tilde{\rho}+\tilde{p}) x}{2 C(x)}\left[\tilde{p}+\frac{2 M(x)}{x^{3}}\right],
$$

which is nothing but the TOV equations in the GR frame, where the mass function satisfies

$$
M_{x}=\frac{\kappa^{2} x^{2}}{2} \tilde{\rho}(x)
$$

Note that the nonrelativistic limit of Eq. (24) does not allow us to directly take $\tilde{p}$ away, since it contains terms in $\rho^{2}$ when moving to the GR frame via the mapping equations (12) and (13), which is our next goal. To this end, upon suitable combinations of these mapping equations, together with Eq. (19), we find in this case the following relations between the energy densities and the pressures on both frames

$$
\begin{gathered}
\tilde{\rho} \approx \frac{\rho-\frac{(\gamma+\delta) \rho^{2}}{2 \kappa^{2}}}{|\hat{\Omega}|^{1 / 2}} \approx \rho+\left(2 \alpha+\frac{\beta}{2}-\frac{\gamma+\delta}{2 \kappa^{2}}\right) \rho^{2}, \\
\tilde{p} \approx \frac{p+\frac{(\gamma+\delta) \rho^{2}}{2 \kappa^{2}}}{|\hat{\Omega}|^{1 / 2}} \approx p+\frac{(\gamma+\delta)}{2 \kappa^{2}} \rho^{2} .
\end{gathered}
$$

On the other hand, we need to incorporate in this analysis the explicit transformation between the radial coordinates in the GR and RBG frames via the fundamental relation (3). Thus, assuming also a static, spherically symmetric line element for the space-time geometry $g_{\mu \nu}$ with a new radial coordinate $r$, using (18) one finds that, in the nonrelativistic approximations employed so far, Eq. (3) yields the result

$$
x^{2}=r^{2}(1-\alpha \rho)
$$

neglecting again both $\rho^{2}$ and $\rho \cdot P$ terms in this approximation.

We are now ready to express (24) in terms of the physical variables. We start with the mass function (25). Using (26) and (28) one finds that the mass function can be integrated as

$$
M(r) \approx M_{0}(r)+\eta(r),
$$

where we have introduced the definition

$$
M_{0}(r)=\frac{\kappa^{2}}{2} \int^{r} d R R^{2} \rho(R),
$$

which is nothing but the GR contribution to the mass, while the additional piece reads (here $\rho^{\prime} \equiv d \rho / d r$ ) $\eta(r)=\frac{\kappa^{2}}{4} \int^{r} d R R^{2} \rho^{2}\left[\alpha\left(1-\frac{r \rho^{\prime}}{\rho}\right)+\beta-\frac{\gamma+\delta}{\kappa^{2}}\right]$,

in which we have neglected again cubic terms in $\rho$ in the integrand. Now, from Eq. (24), using the expression for the mass function (29) alongside the mapping equations particularized to this case, Eqs. (26) and (27), we get

$$
\begin{aligned}
\tilde{p}_{x} \approx & -\frac{r \rho}{2}\left[\left(p+\frac{2 M_{0}}{r^{3}}\right)+\frac{2 M_{0}}{r^{3}}\left(\frac{6 \alpha+\beta}{2}\right) \rho\right. \\
& \left.+\left(\frac{\gamma+\delta}{2 \kappa^{2}}\right) \rho^{2}+\left(1+\frac{3}{2} \alpha \rho\right) \eta(r)\right] .
\end{aligned}
$$

Finally we need to work out the expression on the left-hand side of this equations in terms of the physical variables using again the relation between coordinates in both frames, Eq. (28). After suitably rearranging terms, we arrive to the final result

$$
\begin{aligned}
p_{r} \approx & -\frac{M_{0} \rho}{r^{2}}\left[1+\frac{p r^{3}}{2 M_{0}}+\left(\frac{5 \alpha+\beta-\alpha r^{2} \rho^{\prime} / \rho}{2}\right) \rho\right. \\
& \left.+\frac{(\gamma+\delta) r^{3}}{4 \kappa^{2} M_{0}}\left(1+4 \rho^{\prime} /\left(r \rho^{2}\right)\right) \rho^{2}+\frac{r^{3}}{2 M_{0}}\left(1+\frac{3}{2} \alpha \rho\right) \eta(r)\right] .
\end{aligned}
$$

This equation fully parameterizes the nonrelativistic limit of the RBG family in terms of the coefficients $(\gamma, \delta)$, associated to the expansion of the RBG Lagrangian, $\mathcal{L}_{G}$, and the coefficients $(\alpha, \beta)$, associated to the expansion of the deformation matrix, $\Omega^{\mu}{ }_{\nu}$. In GR, $\alpha=\beta=\gamma=\delta=0$ and neglecting the pressure term in (33) one recovers the Newtonian limit

$$
p_{r}^{\mathrm{GR}}=-\frac{M_{0}(r) \rho(r)}{r^{2}},
$$

from which the well known Lane-Emden equation is derived assuming a polytropic fluid and on top of which studies of nonrelativistic stars are typically carried out.

The bottom line of this result is that all RBGs, no matter the explicit functional dependencies of their Lagrangian densities, have the same qualitative behavior in what concerns their nonrelativistic limit of stellar structure. This means that, once a particular RBG model is given, a simple expansion of its Lagrangian density in (20) and of the deformation matrix in (17) allows us to find the parameters associated to the nonrelativistic regime and, therefore, starting from it different aspects of the physics of nonrelativistic stars can be immediately implemented. To this end, one needs to estimate the relative strength of each term contributing to this equation for any kind of such star. Therefore, we first keep in this expression only those terms linear and quadratic in the energy density and linear in the pressure, so we get 


$$
\begin{aligned}
p_{r} \approx & -\frac{M_{0} \rho}{r^{2}}\left[1+\frac{p r^{3}}{2 M_{0}}+\left(\frac{5 \alpha+\beta-\alpha r^{2} \rho^{\prime} / \rho}{2}\right) \rho\right. \\
& \left.\left.+\frac{(\gamma+\delta) r^{2} \rho^{\prime}}{\kappa^{2} M_{0}}\right)\right] .
\end{aligned}
$$

For a nonrelativistic star typically the pressure is modeled by using a polytropic equation of state [27]

$$
p=K \rho^{\Gamma},
$$

where the polytropic constant $K$ and index $\Gamma=1+1 / n$ with $n>1$ are capable to characterize different types of nonrelativistic stars. ${ }^{3}$ Therefore, the term in $p$ can be typically neglected as compared to those in $\rho$, and in the nonrelativistic limit the approximations $p \ll \rho, r^{3} p \ll M$, $2 M_{0} / r \ll 1$ hold. Therefore, we shall use formula (35) with the constraint above for the discussion on specific examples of RBGs.

\section{A. Quadratic $f(R)$ gravity}

The simplest example of an RBG is given by $f(R)$ theories. Let us consider the well-known quadratic (Starobinsky) $f(R)$ gravity, which is given by the Lagrangian density [68]

$$
f(R)=\frac{1}{2 \kappa^{2}}\left(R+\mu R^{2}\right),
$$

where $\mu$ is a parameter with dimensions of length squared. For this theory, in the metric-affine formalism it turns out that $R=-\kappa^{2} T$ via the corresponding field equations (whose trace implies that $R f_{R}-2 f=\kappa^{2} T$ ), which is the same result as in GR. This allows us to write this Lagrangian in terms of energy density and pressure (in the nonrelativistic limit) as

$$
\mathcal{L}_{G}=\frac{1}{2 \kappa^{2}} f(R) \approx \frac{\rho-3 p}{2}+\frac{\mu \kappa^{2} \rho^{2}}{2}
$$

On the other hand, the deformation matrix in this case reads $\Omega^{\mu}{ }_{\nu}=f_{R} \delta_{\nu}^{\mu}$, where $f_{R} \equiv d f / d R$, which entails a conformal relation between the space-time and auxiliary metrics. From Eqs. (26) and (27), one is equipped with the following relations between energy density and pressure in the GR and RBG frames

$$
\tilde{\rho}=\frac{\rho-\mu \kappa^{2} / 4 \rho^{2}}{\left(1+\epsilon \kappa^{2} \rho\right)^{2}} \approx \rho-\frac{7 \mu \kappa^{2} \rho^{2}}{2},
$$

\footnotetext{
${ }^{3}$ For equations of state $p=(\Upsilon-1) \rho$ with $0<\Upsilon<1$ the pressure term is of order below than the one in $\rho$. However, such equations of interest are not of interest for the physics of these stars and can therefore be neglected in our analysis.
}

$$
\tilde{p}=\frac{p+\mu \kappa^{2} / 4 \rho^{2}}{\left(1+\epsilon \kappa^{2} \rho\right)^{2}} \approx p+\frac{\mu \kappa^{2} \rho^{2}}{2},
$$

which agrees with the results of [69]. With these expressions at hand, and from Eqs. (18) and (20), it is a simple exercise to see that the parameters $\alpha=-2 \mu \kappa^{2}, \beta=0$, $\gamma=-\mu \kappa^{4}, \delta=0$ and thus Eq. (35), neglecting the contribution of the pressure, reads

$$
p_{r}=-\frac{M_{0} \rho}{r^{2}}\left[1-\mu \kappa^{2}\left(5 \rho-r \rho^{\prime}\right)-\frac{\mu \kappa^{2} r^{2} \rho^{\prime}}{M_{0}}\right]
$$

which is the expression for the nonrelativistic limit of quadratic $f(R)$ gravity with the assumptions above.

\section{B. Eddington-inspired Born-Infeld gravity}

Another well known example of a RBG employed in the literature is EiBI gravity, given by the action [70]

$$
S_{\mathrm{EiBi}}=\frac{1}{\epsilon \kappa^{2}} \int d^{4} x\left[\sqrt{\left|g_{\mu \nu}+\epsilon R_{\mu \nu}\right|}-\lambda \sqrt{-g}\right]
$$

where $\epsilon$ is EiBI parameter with dimensions of length squared, and the theory enjoys an effective cosmological constant $\Lambda_{\text {eff }}=\frac{\lambda-1}{\epsilon}$. The astrophysical and cosmological phenomenology for this theory and its many extensions was thoroughly discussed in [11]. The Lagrangian density for EiBI gravity can be conveniently written as

$$
\mathcal{L}_{\mathrm{EiBi}}=\frac{|\hat{\Omega}|^{1 / 2}-\lambda}{\epsilon \kappa^{2}}
$$

while the deformation matrix in this case is implicitly given by the algebraic equation

$$
|\hat{\Omega}|^{1 / 2}\left(\Omega^{\mu}{ }_{\nu}\right)^{-1}=\lambda \delta_{\nu}^{\mu}-\epsilon \kappa^{2} T_{\nu}^{\mu} .
$$

In order to solve it, one inserts the expression of the perfect fluid (10) and upon resolution and comparison with (18) one finds (for asymptotically flat configurations, $\lambda=1$ ) the parameters $(\gamma+\delta)=\epsilon \kappa^{2} / 4$ while from Eq. (43) one gets that $\alpha=-\epsilon \kappa^{2} / 2, \beta=\epsilon \kappa^{2}$. Therefore, upon substitution in Eq. (35), we are left with the result

$$
p_{r}=-\frac{M_{0} \rho}{r^{2}}-\frac{\epsilon \kappa^{2} \rho \rho^{\prime}}{4}+\frac{\epsilon \kappa^{2} M_{0}\left(3-r \rho^{\prime} / \rho\right) \rho}{4 r^{2}},
$$

and then by imposing that $r \gg 2 M_{0}$ the last term can be neglected and one ends up with the expression reported in Ref. [71].

\section{CONCLUSION AND DISCUSSION}

The physics of nonrelativistic stars, though comparatively much less explored than their relativistic counterparts 
(neutron stars), nonetheless offers a viable opportunity to test the predictions and consistency of modified gravity theories with astrophysical observations. It is so because of less severe unknowns on nongravitational aspects of these stars, since those can be well modeled from a phenomenological perspective via observations. Though fully reliable predictions can only be obtained after incorporating all the physics known to play a relevant role in these objects and require the use of numerical simulations, simple analytical models offer reasonable approximations to some of the most relevant global aspects of the physics of nonrelativistic stars.

The above scenario is particularly relevant for those modified theories of gravity yielding modifications of the gravitational field inside astrophysical sources while leaving the vacuum dynamics unaffected (so as to pass solar system experiments). This is a property naturally hold by the family of Ricci-based gravities considered in this work, without any need of implementing screening/chameleon mechanisms [38]. Elaborating from the field equations of these theories framed in a representation in terms of an auxiliary metric (its Einstein frame), we have used a recently found mapping between these equations and those of GR, by which both theories are coupled to the same kind of fields but with different functional dependencies on their Lagrangian densities, the explicit form of which is obtained once a specific RBG theory is given. Here we have shown that this mapping allows formal manipulations of the corresponding field equations simplifying the process to obtain the corresponding nonrelativistic limit of the stellar structure equations, somewhat paralleling the well-known duality of the Einstein/Jordan frames of the metric $f(R)$ case. Via this process, the main result of the present work is to show that all RBGs enjoy the same nonrelativistic equation, parameterized in terms of four constants associated to the expansion in powers of the energy-momentum of the matter fields of the gravitational Lagrangian and of the deformation matrix realizing the transformation between the space-time and auxiliary metrics.

Many recent works in the field have shown the viability of the nonrelativistic stellar limit of modified theories of gravity to obtain specific predictions of these theories that can be accessible via astronomical observations. Prominent among them are the limiting masses between different kinds of dwarf and main-sequence stars [31-42,56]. These limits depend not only on the combination of astrophysical and nuclear physics modeling, but also on the underlying nonrelativistic equation employed, where modifications to the latter may slightly alter such limits and therefore yield new predictions and/or allow us to place constraints on the parameters of specific modified gravity theories. On the other hand, some recent challenges to these limiting masses within GR such as the existence of super-Chandrasekhar white dwarfs could be explained via modified nonrelativistic equations beyond GR [72]. The RBG family of theories and its nonrelativistic limit introduced here therefore offer a suitable playground to keep exploring the predictions of theories when supplemented with suitable equations of state and incorporating the additional physical elements needed to reliably model dwarf and mainsequence stars. This would allow us to place more stringent constraints on the astrophysical viability of these theories as compared to GR.

\section{ACKNOWLEDGMENTS}

D. R.G. is funded by the Atracción de Talento Investigador programme of the Comunidad de Madrid (Spain) No. 2018-T1/TIC-10431, and acknowledges further support from the Ministerio de Ciencia, Innovación y Universidades (Spain) Project No. PID2019-108485 GBI00/AEI/10.13039/501100011033, and the FCT Projects No. PTDC/FIS-PAR/31938/2017 and No. PTDC/FISOUT/29048/2017. A. W. is supported by the EU through the European Regional Development Fund CoE program TK133 "The Dark Side of the Universe". This work is supported by the Spanish Projects No. FIS2017-84440-C21-P (MINECO/FEDER, EU), No. PROMETEO/2020/079 (Generalitat Valenciana), and No. i-COOPB20462 (CSIC), and by the Edital 006/2018 PRONEX (FAPESQ-PB/ CNPQ, Brazil, Grant No. 0015/2019). This article is based upon work from COST Action CA18108, supported by COST (European Cooperation in Science and Technology). D. R. G. and A. W. thank the Department of Physics and IFIC of the University of Valencia for their hospitality during different stages of the elaboration of this work.
[1] B. P. Abbott et al. (LIGO Scientific and Virgo Collaborations), Phys. Rev. Lett. 116, 061102 (2016).

[2] B. P. Abbott et al. (LIGO Scientific and Virgo Collaborations), Phys. Rev. Lett. 119, 161101 (2017).

[3] K. Akiyama et al. (Event Horizon Telescope Collaboration), Astrophys. J. 875, L1 (2019).

[4] C. M. Will, Living Rev. Relativity 17, 4 (2014).
[5] P. Bull et al., Phys. Dark Universe 12, 56 (2016).

[6] G. J. Olmo, Int. J. Mod. Phys. D 20, 413 (2011).

[7] A. De Felice and S. Tsujikawa, Living Rev. Relativity 13, 3 (2010).

[8] S. Capozziello and M. De Laurentis, Phys. Rep. 509, 167 (2011).

[9] T. Clifton, P. G. Ferreira, A. Padilla, and C. Skordis, Phys. Rep. 513, 1 (2012). 
[10] S. Nojiri, S. D. Odintsov, and V. K. Oikonomou, Phys. Rep. 692, 1 (2017).

[11] J. B. Jimenez, L. Heisenberg, G. J. Olmo, and D. RubieraGarcia, Phys. Rep. 727, 1 (2018).

[12] L. Heisenberg, Phys. Rep. 796, 1 (2019).

[13] V. Cardoso, S. Hopper, C. F. B. Macedo, C. Palenzuela, and P. Pani, Phys. Rev. D 94, 084031 (2016).

[14] V. Cardoso and P. Pani, Nat. Astron. 1, 586 (2017).

[15] R. A. Konoplya, Z. Stuchlík, and A. Zhidenko, Phys. Rev. D 99, 024007 (2019).

[16] E. Berti, K. Yagi, H. Yang, and N. Yunes, Gen. Relativ. Gravit. 50, 49 (2018).

[17] J. M. Ezquiaga and M. Zumalacárregui, Phys. Rev. D 102, 124048 (2020).

[18] S. E. Gralla, D. E. Holz, and R. M. Wald, Phys. Rev. D 100, 024018 (2019).

[19] R. Carballo-Rubio, F. Di Filippo, S. Liberati, and M. Visser, Phys. Rev. D 98, 124009 (2018).

[20] M. Wielgus, J. Horak, F. Vincent, and M. Abramowicz, Phys. Rev. D 102, 084044 (2020).

[21] M. Afrin, R. Kumar, and S. G. Ghosh, Mon. Not. R. Astron. Soc. 504, 5927 (2021).

[22] H. C. D. L. Junior, L. C. B. Crispino, P. V. P. Cunha, and C. A. R. Herdeiro, Phys. Rev. D 103, 084040 (2021).

[23] J. Peng, M. Guo, and X. H. Feng, arXiv:2102.05488.

[24] E. Berti et al., Classical Quantum Gravity 32, 243001 (2015).

[25] J. M. Lattimer and M. Prakash, Science 304, 536 (2004).

[26] A. Burrows and J. Liebert, Rev. Mod. Phys. 65, 301 (1993).

[27] N. K. Glendenning, Compact Stars: Nuclear Physics, Particle Physics, and General Relativity, 2nd ed., Astronomy and Astrophysics Library (Springer-Verlag, New York, 2000).

[28] G. J. Olmo, D. Rubiera-Garcia, and A. Wojnar, Phys. Rep. 876, 1 (2020).

[29] R. Saito, D. Yamauchi, S. Mizuno, J. Gleyzes, and D. Langlois, J. Cosmol. Astropart. Phys. 06 (2015) 008.

[30] S. Chandrasekhar, Mon. Not. R. Astron. Soc. 95, 207 (1935).

[31] I. D. Saltas, I. Sawicki, and I. Lopes, J. Cosmol. Astropart. Phys. 05 (2018) 028.

[32] R. K. Jain, C. Kouvaris, and N. G. Nielsen, Phys. Rev. Lett. 116, 151103 (2016).

[33] S. Banerjee, S. Shankar, and T. P. Singh, J. Cosmol. Astropart. Phys. 10 (2017) 004.

[34] A. Wojnar, https://doi.org/10.1142/S0219887821400065, arXiv:2012.13927.

[35] I. H. Belfaqih, H. Maulana, and A. Sulaksono, arXiv: 2104.11774.

[36] J. Sakstein, Phys. Rev. Lett. 115, 201101 (2015).

[37] J. Sakstein, Phys. Rev. D 92, 124045 (2015).

[38] M. Crisostomi, M. Lewandowski, and F. Vernizzi, Phys. Rev. D 100, 024025 (2019).

[39] A. S. Rosyadi, A. Sulaksono, H. A. Kassim, and N. Yusof, Eur. Phys. J. C 79, 1030 (2019).

[40] A. Wojnar, Phys. Rev. D 102, 124045 (2020).

[41] M. Benito and A. Wojnar, Phys. Rev. D 103, 064032 (2021).

[42] A. Wojnar, Phys. Rev. D 103, 044037 (2021).
[43] S. Bahamonde and J. G. Valcarcel, Eur. Phys. J. C 81, 495 (2021).

[44] V. I. Afonso, G. J. Olmo, and D. Rubiera-Garcia, Phys. Rev. D 97, 021503 (2018).

[45] D. Rubiera-Garcia, Int. J. Mod. Phys. D 29, 2041007 (2020).

[46] C. Bejarano, G. J. Olmo, and D. Rubiera-Garcia, Phys. Rev. D 95, 064043 (2017).

[47] S. D. Odintsov, G. J. Olmo, and D. Rubiera-Garcia, Phys. Rev. D 90, 044003 (2014).

[48] A. D. I. Latorre, G. J. Olmo, and M. Ronco, Phys. Lett. B 780, 294 (2018).

[49] A. Delhom, V. Miralles, and A. Peñuelas, Eur. Phys. J. C 80, 340 (2020).

[50] J. B. Jiménez, A. Delhom, G. J. Olmo, and E. Orazi, arXiv: 2104.01647.

[51] J. Antoniadis et al., Science 340, 1233232 (2013).

[52] D. A. Howell et al. (SNLS Collaboration), Nature (London) 443, 308 (2006).

[53] I. Hachisu, M. Kato, H. Saio, and K. Nomoto, Astrophys. J. 744, 69 (2012).

[54] U. Das and B. Mukhopadhyay, Phys. Rev. Lett. 110, 071102 (2013).

[55] E. Y. Hsiao et al., Astrophys. J. 900, 140 (2020).

[56] G. J. Olmo, D. Rubiera-Garcia, and A. Wojnar, Phys. Rev. D 100, 044020 (2019).

[57] L. A. Hillenbrand and R. J. White, Astrophys. J. 604, 741 (2004).

[58] A. W. Mann et al., Astrophys. J. 871, 63 (2019).

[59] V. I. Afonso, C. Bejarano, J. B. Jimenez, G. J. Olmo, and E. Orazi, Classical Quantum Gravity 34, 235003 (2017).

[60] J. Beltrán Jiménez and A. Delhom, Eur. Phys. J. C 79, 656 (2019).

[61] J. B. Jiménez and A. Delhom, Eur. Phys. J. C 80, 585 (2020).

[62] We thank José Beltrán Jiménez for enforcing us the viewpoint that RBGs can be seen as GR with new nonlinear couplings in the matter fields, which has led us to new insights on these theories.

[63] V. I. Afonso, G. J. Olmo, E. Orazi, and D. Rubiera-Garcia, Eur. Phys. J. C 78, 866 (2018).

[64] V. I. Afonso, G. J. Olmo, E. Orazi, and D. Rubiera-Garcia, Phys. Rev. D 99, 044040 (2019).

[65] M. Guerrero, G. Mora-Pérez, G. J. Olmo, E. Orazi, and D. Rubiera-Garcia, J. Cosmol. Astropart. Phys. 07 (2020) 058.

[66] G. Raposo, P. Pani, M. Bezares, C. Palenzuela, and V. Cardoso, Phys. Rev. D 99, 104072 (2019).

[67] J. B. Jiménez, D. De Andrés, and A. Delhom, Classical Quantum Gravity 37, 225013 (2020).

[68] A. A. Starobinsky, Phys. Lett. 91B, 99 (1980).

[69] A. Mana, L. Fatibene, and M. Ferraris, J. Cosmol. Astropart. Phys. 10 (2015) 040.

[70] M. Banados and P. G. Ferreira, Phys. Rev. Lett. 105, 011101 (2010).

[71] P. Pani, V. Cardoso, and T. Delsate, Phys. Rev. Lett. 107, 031101 (2011).

[72] H. Wei and Z.X. Yu, arXiv:2103.12696. 